\title{
Gabor wavelets on the sphere
}

\section{Laurent Demanet, Pierre Vandergheynst}

Laurent Demanet, Pierre Vandergheynst, "Gabor wavelets on the sphere," Proc. SPIE 5207, Wavelets: Applications in Signal and Image Processing X, (13 November 2003); doi: 10.1117/12.506436

SPIE Event: Optical Science and Technology, SPIE's 48th Annual Meeting, 2003, San Diego, California, United States 


\title{
Gabor Wavelets on the Sphere
}

\author{
Laurent Demanet ${ }^{a}$ and Pierre Vandergheynst ${ }^{b}$ \\ ${ }^{a}$ Applied and Computational Mathematics, California Institute of Technology, MC 217-50, \\ Pasadena CA 91125, USA \\ ${ }^{b}$ Signal Processing Laboratory, Swiss Federal Institute of Technology (EPFL), CH 1015, \\ Lausanne, Switzerland
}

\begin{abstract}
We propose a construction of directional - or Gabor - continuous wavelets on the sphere. We provide a criterion to measure their angular selectivity. We finally discuss implementation issues and potential applications. The code for the spherical wavelet transform is avalaible in the YAWTB Matlab toolbox, http://www.yawtb.be.tf.
\end{abstract}

Keywords: Directional Wavelets; Sphere; Conformal dilation; Angular Resolving Power

\section{INTRODUCTION}

The 2-D Euclidean Continuous Wavelet Transform (CWT) is often discussed in the special case when the wavelet is isotropic. In that case the parameter space consists of translations (2 parameters) and dilations (one parameter). The purpose of the isotropic CWT is to perform bandpass filtering at different scales. Geometric aspects can be introduced in the transform using a fourth parameter, orientation. If chosen appropriately, the wavelet can be made to correlate preferentially to features oriented in a specific direction, like straight lines or elongated brush strokes in a picture. In that case the wavelet is said to be anisotropic or directional. ${ }^{1}$ The concept of filtering at different scales and orientations was first introduced in the vision community under the name of oriented Gabor filters.

The potential for multiscale geometric transforms, whether decimated or redundant, is largely unexplored in today's applications. Orientation selectivity alone is a desirable tool in some image analysis and recognition tasks, but there is more to the story :

- Truly sparse image or video coding cannot be obtained without taking into account some geometric features of real-life scenes, e.g., the presence of object edges or texture. It has been shown ${ }^{2}$ that curvelets (directional wavelets with a specific scale-dependent angular sampling scheme) are very promising at approximating sharp discontinuities along broken lines or curves. Such are the contours of any object in a picture. The achieved asymptotic rate of decay of the error of the best $M$-term approximation using curvelets is superior to that using non-oriented wavelets. The efficiency of anisotropic wavelet-like atoms at compressing object contours has also been successfully demonstrated at very low bit rates and using a matching pursuit implementation ${ }^{3}{ }^{4}$

- Standard multiscale ideas currently dominate the field of computational partial differential equations. A numerical solution is usually refined on successive grids at different scales. Yet in several dimensions much could be gained from also 'refining' in angle. It has recently been proved ${ }^{5}$ that the same curvelets as above provide correct decomposition of phase-space for linear hyperbolic equations (like the wave equation). Oscillatory integrals arising from these problems admit a well-structured and sparse representation in terms of curvelets, as opposed to isotropic wavelets. This paves the way to fast solvers for acoustic and electromagnetic wave propagation in heterogeneous media.

E-mail: demanet@acm.caltech.edu, pierre.vandergheynst@epfl.ch 
In this paper we are concerned with extending the concept of directional wavelets, thus also Gabor filters and curvelets, to the spherical case. The only surprise is that the notion of direction on the sphere is itself not well-defined. We have no ambition of addressing the delicate task of designing a Discrete Wavelet Transform (DWT) on the sphere, as initiated by Schröder and Sweldens. ${ }^{6}$

Section 2 recalls the construction of spherical continuous wavelets as introduced by Antoine et al. in ${ }^{7}{ }^{8}$ Other authors define the spherical CWT slightly differently, see ${ }^{9}$ and references therein. Their setting is however not the most appropriate for localized or anisotropic wavelets. The design of truly directional wavelets on the sphere is discussed in section 3. Any Euclidean directional wavelet can be projected onto the sphere without losing its properties. Section 4 presents a very basic algorithm to invert the CWT, provided an appropriate wavelet is chosen. This method of reconstruction, called scale-angle packets, makes the CWT particularly competitive whenever redundancy of the transform is not an issue. Finally, some applications are discussed in section 5.

\section{DEFINITIONS}

\subsection{Generalities}

Let us start by recalling some of the definitions related to the continuous spherical wavelet transfom. The interested readers should refer to ${ }^{8}$ for a detailed discussion.

Denote by $\omega=(\theta, \phi)$ a point on the 2 -sphere $S^{2}$. A function $\Psi \in L^{2}\left(S^{2}, \sin \theta d \theta d \phi\right)$ undergoes the following basic transformations,

- Rigid rotations Let $g$ be an element of the group of rotations $S O(3)$ acting on $S^{2}$. Then $\Psi$ is rigidly transported on the sphere according to

$$
R_{g} \Psi(\omega)=\Psi\left(g^{-1} \omega\right)
$$

- Conformal dilations Let $a>0$. Then $\Psi$ centered at the north pole can be dilated or contracted to have scale $a$ as follows,

$$
D_{a} \Psi(\omega) \equiv \Psi_{a}(\omega)=\lambda(a, \theta)^{1 / 2} \Psi\left(\omega_{a}\right)
$$

where $\omega_{a} \equiv\left(\theta_{a}, \phi\right)$ and $\tan \left(\theta_{a} / 2\right)=a \tan (\theta / 2)$. The factor

$$
\lambda(a, \theta)=\frac{4 a^{2}}{\left[\left(a^{2}-1\right) \cos \theta+\left(a^{2}+1\right)\right]^{2}}
$$

is a 'cocyle' and makes the dilation unitary. As can be checked this transformation preserves angles, hence the name conformal.

The continuous wavelet transform (CWT) of a function $f \in L^{2}\left(S^{2}\right)$ for a given $\Psi$ is defined as

$$
W_{\Psi} f(g, a)=\left\langle f, R_{g} \Psi_{a}\right\rangle=\int_{S^{2}} d \omega f(\omega) \overline{\Psi_{a}\left(g^{-1} \omega\right)} .
$$

The measure $d \omega$ is the usual Lebesgue measure on the sphere, $d \omega=\sin \theta d \theta d \phi$. The parameter space $S O(3) \times \mathbb{R}_{*}^{+}$ of the CWT has 4 dimensions. The function $\Psi$ is not arbitrary ; in order to be called a wavelet it should also satisfy the admissibility condition

$$
\int \frac{\Psi(\theta, \phi)}{1+\cos \theta} \sin \theta d \theta d \phi=0
$$

which morally amounts to a zero-mean condition. In that case the CWT can be inverted on its range by a double integral in $g$ and $a$, up to a nice multiplier operator.

Unlike in the Euclidian case, the notion of dilation on a compact manifold like the sphere is not obvious. Our choice is rooted in group-theoretical considerations. The action of rotations and dilations on a function on the sphere is a representation of (a subgroup of) the Lorentz group $S O_{0}(3,1)$, which acts conformally on the sphere. The CWT is named 'generalized coherent states' in that setting. ${ }^{10}$ 
The conformal dilation also has a nice geometric interpretation. Let us introduce the stereographic projection $\Pi: S^{2} \rightarrow \mathbb{R}^{2}$ as taking a point $\omega$ on the sphere to the point $\Pi \omega$ on the tangent plane to the north pole, colinear with $\omega$ and the south pole. Acting on functions in $L^{2}\left(S^{2}\right)$, the stereographic projection can be defined through

$$
\Pi f(r, \phi)=\frac{4}{4+r^{2}} f\left(2 \operatorname{atan} \frac{r}{2}, \phi\right),
$$

or

$$
\Pi^{-1} f(\theta, \phi)=\frac{2}{1+\cos \theta} f\left(2 \tan \frac{\theta}{2}, \phi\right) .
$$

It is easy to see that $\Pi$ is a unitary map from $L^{2}\left(S^{2}, \sin \theta d \theta d \phi\right)$ to $L^{2}\left(\mathbb{R}^{2}, r d r d \phi\right)$. The conformal dilation $D_{a}$ can actually be seen as a Euclidian dilation $d_{a}$ in the tangent plane at the north pole, through conjugation by $\Pi$,

$$
D_{a} f=\Pi^{-1} d_{a} \Pi f
$$

where $d_{a} f(x)=\frac{1}{a} f\left(\frac{x}{a}\right)$. The cocycle factor (1) can be recovered from expanding the above formula.

\subsection{Isotropic vs. Anisotropic Wavelets}

The wavelet $\Psi$ is said to be isotropic or zonal if it does not depend on $\phi$ when centered at the north pole. In that case the rotation parameter corresponding to rotations around the north pole is redundant and can be omitted. The two remaining rotation parameters correspond to a position on the sphere, in accordance with the isomorphism $S^{2} \simeq S O(3) / S O(2)$. The parameter space of the transform is therefore reduced to $S^{2} \times \mathbb{R}_{*}^{+}$. As shown in $^{8}$ p. 190, the CWT is for every $a$ a spherical convolution,

$$
W_{\Psi} f(\omega, a)=\int d \omega^{\prime} f\left(\omega^{\prime}\right) \overline{\Psi_{a}\left(\omega \cdot \omega^{\prime}\right)}
$$

It corresponds to filtering the signal $f$ at different scales.

For general $\Psi$, all three rotation parameters are relevant. We can interpret $g \in S O(3)$ as $g=(\omega, \chi)$, where $\omega \in S^{2}$ is a position on the sphere and $\chi \in S O(2)$ is an angle of rotation*. Recall that the CWT is expected to realize filtering at different scales $(a)$ and angles $(\chi)$. It is written as

$$
W_{\Psi} f(\omega, \chi, a)=\int d \omega^{\prime} f\left(\omega^{\prime}\right) \overline{R_{\chi} \Psi_{a}\left([\omega]^{-1} \omega^{\prime}\right)} .
$$

The notation $R_{\chi} \Psi$ refers to the inital rotation of an angle $\phi$ of the wavelet around the north pole. The map [.] : $S^{2} \rightarrow S O(3)$ is called a section in group theory. Its choice is arbitrary but crucial. It determines the orientation in which the wavelet will reside after the transport by $[\omega]$. It can be depicted as a map from the sphere to a field of tangent vectors of unit length defined on it. The angle $\chi$ only makes sense as the orientation relative to that vector field of reference. In other words, we are facing the problem of choosing a global direction on the sphere. This is topologically impossible : any section must have at least one singular point ${ }^{\dagger}$.

To fix ideas, let us consider the parameterization of $S O(3)$ given by the Euler angles. If we denote by $O x, O y, O z$ the three cartesian axes, any rotation $g=(\alpha, \beta, \chi)$ of $S O(3)$ can be decomposed as (1) a rotation of angle $\chi$ around $O z,(2)$ a second rotation of angle $\beta$ around $O x$ and (3) a last rotation of angle $\alpha$ around $O z$ again $^{\ddagger}$. We identify $\omega \in S^{2}$ as $(\alpha, \beta)$ and $[\omega] \in S O(3)$ as $(\alpha, \beta, 0)$. Pictorially, this choice of section corresponds to tangent vectors pointing all, say, to the east. The two singular points are the north and south poles. The notion of direction relative to that vector field is interesting and intuitive near the equator but fails at the poles : the cardinal points are not defined there. Other parameterizations than the Euler angles can of course be chosen, like the 'pitch-roll-yaw' convention.

As a conclusion, the values of the spherical CWT corresponding to a given angle $\chi$ can accomodate for the idea of oriented filtering, but the notion of direction itself is not clear. It is always relative to some arbitrary

\footnotetext{
${ }^{*}$ A geometer would do it by means of the natural projection from the fiber bundle $S O(3)$ onto the base manifold $S^{2}$. ${ }^{\dagger}$ One cannot comb a perfectly spherical porcupine! One could in the case of $S^{1}$ and the hyperspheres $S^{3}$ and $S^{7}$.

${ }^{\ddagger}$ The rotations are to be considered in the given order. Our convention is that the axes are unaffected by the rotations
} 
reference - the cardinal points in the case of the Euler angles. We will see in the next section how to choose the wavelet $\Psi$ in order to perform efficient directional filtering.

For practical purposes, the transform (6) should be discretized. The open source YAWTB toolbox contains among others an implementation of the directional CWT on the sphere, and is downloadable from

http://www . yawtb.be.tf

The current version samples signals on straightforward rectangular grids in $\theta$ and $\phi$ and makes use of the Euler angles as explained above. It is computed in $O\left(M N^{3} \log N\right)$ operations using a Fast Fourier Transform in the longitudinal coordinate $\phi$, where $M$ is the number of angles and scales considered, and the total number of data points is $O\left(N^{2}\right)$. Unlike in the isotropic case, the directional CWT is not a spherical convolution and has no obvious expression in terms of spherical harmonics coefficients. See however ${ }^{11}$ for a solution in this direction.

\section{PAINLESS DESIGN OF DIRECTIONAL SPHERICAL WAVELETS}

We have seen in the previous section how to construct the CWT on the sphere, but little has been said so far concerning the choice of the wavelet $\Psi$. We are interested in finding a criterion for directionality. For a 2-D Euclidean wavelet $\psi$, directional selectivity is easily expressed in terms of the Fourier transform of $\psi$ : it should be essentially supported in a cone with apex at the origin. The narrower the cone the better the angular selectivity, at the expense of a larger spatial support.

The Fourier space of the sphere is however very peculiar. It is discrete and made of coefficients corresponding to the spherical harmonics $Y_{l}^{m}$. Unlike in the flat case, the action of rotations on functions on the sphere does not have a straightforward expression in terms of Fourier coefficients. This makes the Euclidean criterion particularly hard to generalize to the spherical case.

We will not worry too much about this obstruction. The intuition of what a directional wavelet should be is clear : at small scale the sphere is locally flat and we can therefore apply the Euclidean test there. We define a spherical wavelet $\Psi$ as being directional if, when centered at the north pole, its stereographic projection $\Pi \Psi$ on the tangent plane is directional in the Euclidean sense. The stereographic projection was discussed in the previous section. It is then dilated at larger scales using the conformal spherical dilation.

Natural candidates as directional wavelets on the sphere are therefore the inverse stereographic projection of any directional wavelet in the plane, $\Psi=\Pi^{-1} \psi$. Note that $\Pi$ and $\Pi^{-1}$ both preserve the admissibility (3) of the wavelets. There is no ambiguity in the choice of scale at which the wavelet should be inverse-projected since the conformal dilation is itself defined in the tangent plane. For example, the Morlet or Gabor wavelet in the plane,

$$
\psi_{M}(x)=e^{i k_{0} \cdot x} e^{-|x|^{2}}
$$

becomes

$$
\Psi_{M}(\theta, \phi)=\frac{e^{i\left|k_{0}\right| \tan (\theta / 2) \cos \left(\phi_{0}-\phi\right)} e^{-(1 / 2) \tan ^{2}(\theta / 2)}}{1+\cos \theta}
$$

on the sphere, where $\phi_{0}$ is the argument of $k_{0}$. The Morlet wavelet becomes (numerically) admissible only when $\left|k_{0}\right|$ is large, say $\left|k_{0}\right|=6$. The real part of this function is represented in Fig. 1 and 2 for various values of the scale and rotation parameters. More illustrations of the spherical Morlet wavelet at work can be found in. ${ }^{8}$

This criterion for directionality makes perfect sense and is the most natural in our setting. Consider a prototypical oriented feature going through the north pole : the characteristic function of longitudinal angles ranging between 0 and $\pi$. Call it $f$. According to (4), the projection $\Pi f$ is a half-dome, supported on, say, the right half-plane. It is also a highly oriented feature in the tangent plane. Take $\psi(r, \phi)$ a directional wavelet in the plane, centered at the origin. Denote $R_{\chi} \psi(r, \phi)=\psi(r, \phi-\chi)$. The decay of the angular correlation

$$
K(\chi)=\left|\left\langle R_{\chi} \psi, \Pi f\right\rangle_{L^{2}\left(\mathbb{R}^{2}\right)}\right|
$$




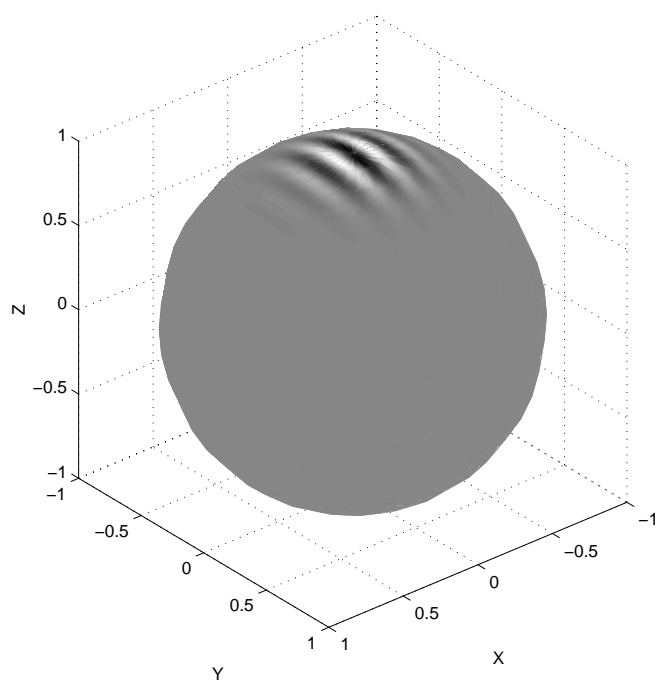

(a)

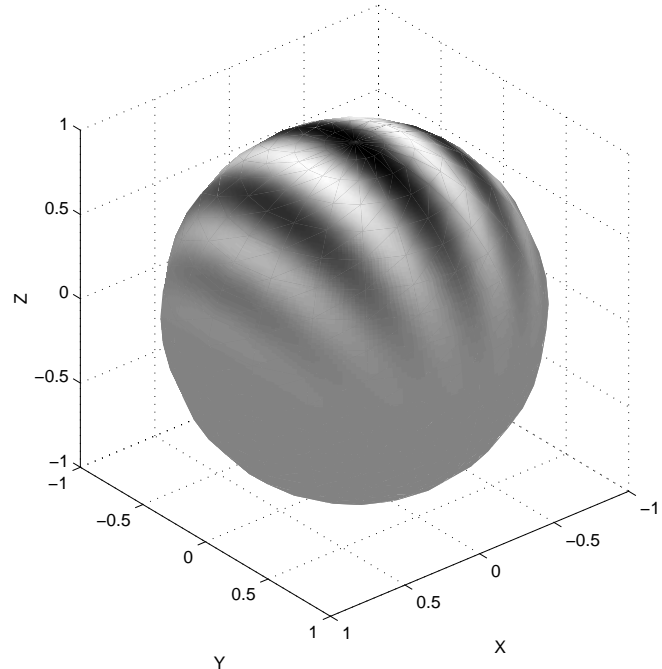

(b)

Figure 1. Real part of the spherical Morlet wavelet at scale (a) $a=0.03$ and (b) $a=0.3$.

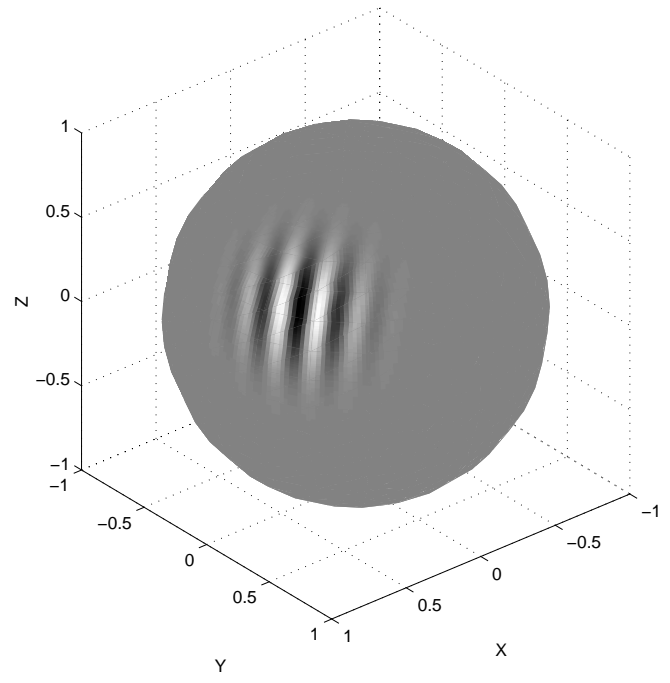

(a)

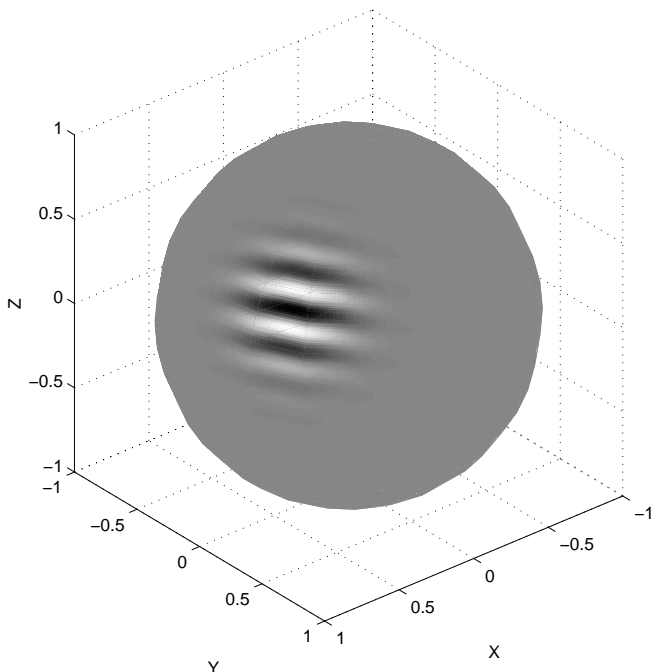

(b)

Figure 2. Real part of the spherical Morlet wavelet at scale $a=0.03$ and centered at $(\pi / 3, \pi / 3)$. (a) $\chi=0$ and (b) $\chi=\pi / 2$.

as the misorientation angle $\chi$ increases indicates how good a directional wavelet $\psi$ is. The faster the decay of $K(\chi)$ away from $\chi=0$, the more sensitive $\psi$. But, according to the aforementioned unitarity of the stereographic projection, we also have

$$
K(\chi)=\left|\left\langle R_{\chi}\left(\Pi^{-1} \psi\right), f\right\rangle_{L^{2}\left(\mathbb{S}^{2}\right)}\right| .
$$

This indicates that $\Pi^{-1} \psi$ is as good a directional wavelet on the sphere as $\psi$ is a directional wavelet in the plane.

Let us now show why any other dilation than the conformal dilation could be disastrous in terms of directional performance at large scale. A Euclidean dilation obviously does not alter the directional power of a planar wavelet 
since it does not modify the cone in which the Fourier transform of the wavelet lies. But how much does a spherical dilation inhibit sensitivity to directions on the sphere ? According to the definitions,

$$
K_{a}(\chi)=\left|\left\langle R_{\chi} d_{a} \psi, \Pi f\right\rangle_{L^{2}\left(\mathbb{R}^{2}\right)}\right|=\left|\left\langle R_{\chi} D_{a}\left(\Pi^{-1} \psi\right), f\right\rangle_{L^{2}\left(\mathbb{S}^{2}\right)}\right| .
$$

The behaviour of the angular correlation is the same as in the planar case, independently of scale. Any other choice of nonconformal dilation $\tilde{D}_{a}$ on the sphere would have resulted in some corresponding pseudo-dilation $\tilde{d}_{a}=\Pi \tilde{D}_{a} \Pi^{-1}$ in the tangent plane. Then $\tilde{d}_{a}$ itself would be nonconformal, since $\Pi$ and $\Pi^{-1}$ are conformal mappings. This will lead to a leakage of the Fourier transform of $\tilde{d}_{a} \psi$ outside of its cone at large scales. Not respecting the geometry imposed by the stereographic projection is dangerous ; it can only impair the directional capabilities of the wavelet.

The observations made in the above paragraphs are instructive and correct but the decay of the angular correlation is not a sufficient indicator of the directional performance of any given wavelet. We forgot to take into account those wavelets that would not be exactly centered on the oriented feature, in our example centered off the north pole. For this purpose, let us define the Angular Resolving Power (ARP) of $\Psi$ at scale $a$ as

$$
\mathrm{ARP} \equiv\left\langle S_{\Psi, a}(\chi)\right\rangle_{\Psi_{a}}=\left\langle\Psi_{a}, S_{\Psi, a}(\chi) \Psi_{a}\right\rangle
$$

where $S_{\Psi, a}(\chi)$ is not simply a rotation but an operator of partial reconstruction,

$$
S_{\Psi, a}(\chi) f(\omega)=\int_{S^{2}} d \omega^{\prime}\left\langle f, R_{\chi} \Psi_{a}\left(\left[\omega^{\prime}\right]^{-1} \omega\right)\right\rangle R_{\chi} \Psi_{a}\left(\left[\omega^{\prime}\right]^{-1} \omega\right) .
$$

The ARP is somewhat similar to the angular correlation $K_{a}(\chi):$ the sharper the peak at zero and the thinner the tails the more directional the wavelet. Intuitively, that is because a directional wavelet itself is an oriented object. The test of the ARP is not only necessary but also sufficient. Since the ARP still depends on the choice of global direction on the sphere through the map [.] (cfr. section 2), we should take $\Psi_{a}$ to be centered in a region where testing directions makes the most sense. For the Euler angles this is near the equator. Also, the ARP does not require to choose a trial oriented singularity to correlate the wavelet to. It can be evaluated on a computer directly from its definition. The ARP is useful not only to compare different wavelets, but also to compare the same wavelet at different scales. Fig. 3 shows how the ARP deteriorates as the scale $a$ of the Morlet wavelet increases. The angular correlation $K_{a}(\chi)$ would fail to show such a phenomenon, as argued previously.

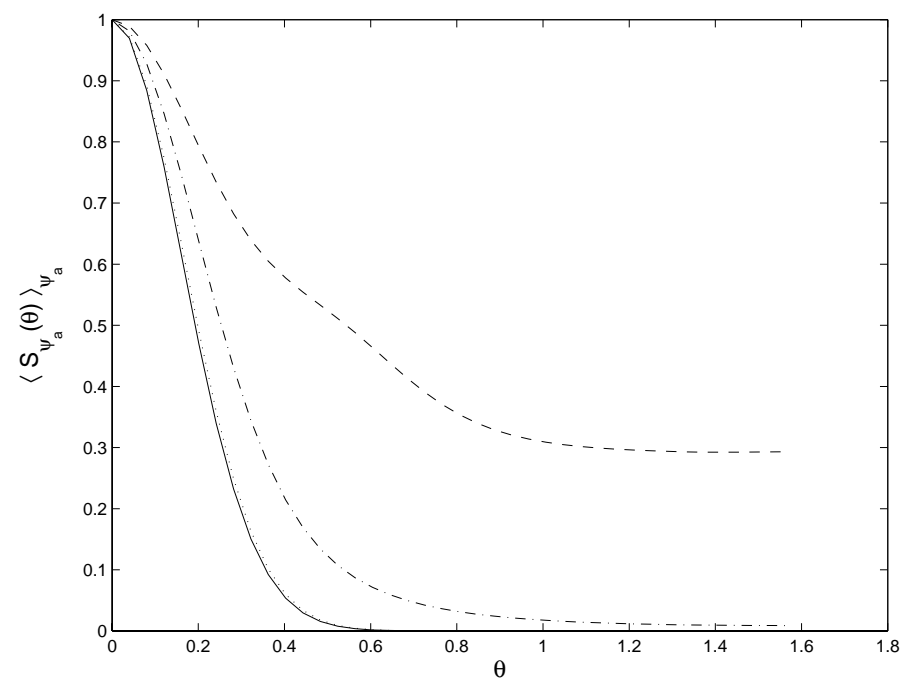

Figure 3. Angular Resolving Power of the spherical Morlet wavelet for various values of the scale parameter : $a=0.03$ (plain), $a=0.1$ (dotted), $a=0.3$ (dashed-dotted) and $a=1$ (dashed).

As a conclusion, the stereographic projection, the conformal dilation and the notion of directional filtering all agree and fit well together. Testing directions is preferably a small-scale procedure, of course, in order to avoid 
the singular points of the global direction. But examination of what to expect at larger scales gives another confirmation that the CWT on the sphere benefits from being formulated using a conformal dilation.

\section{INVERSION TAKES ONE LINE OF CODE}

The inversion formula for the anisotropic CWT on the sphere would be a triple integral in $\omega, a$ and $\chi$, see. $^{7}$ Discretizing these integrals by means of a quadrature formula is a daunting task requiring the computation of way too many values of the transform. Moreover, the continuous formula would not perfectly reconstruct a function $f$, but in general distort it in a nice way ${ }^{\S}$. This is perhaps tolerable in mathematics, but would require the user to run an iterative inversion procedure like conjugate gradients in order to obtain the true inverse up to a good degree of accuracy.

The simple approach we introduce next is not based on the continuous reproducing formula, but rather on a 'telescoping sum' of scale-angle packets.

Take an arbitrary set of scales $0<\ldots<a_{j+1}<a_{j}<\ldots<a_{0}$. The canonical choice would be $a_{j}=2^{-j}$. Choose an isotropic bump function $\varphi(x)$ for $x \in \mathbb{R}^{2}$ such that $\frac{1}{a_{j}^{2}} \varphi\left(\frac{x}{a_{j}}\right)$ defines by convolution an approximation of the identity as $j \rightarrow \infty$. For instance, a gaussian does that just fine. Next, build a smooth partition of unity $w_{l}(\phi)=w\left(\phi-l \phi_{0}\right)$ on the circle, in the sense that $\sum_{l} w_{l}(\phi)=1$. Choose the window $w$ to be (up to smooth tails) the indicator function of an appropriate angular interval. We are now in a position to define a collection of directional wavelets in the plane through their Fourier transform (denoted by a hat),

$$
\hat{\psi}_{j l}(\xi)=\left(\hat{\varphi}\left(a_{j} \xi\right)-\hat{\varphi}\left(a_{j-1} \xi\right)\right) w_{l}(\phi), \quad j \geq 1 .
$$

As is easy to check, these wavelets are admissible, localized, smooth and directional. The missing information at large scales is provided by the 'scaling function'

$$
\hat{\psi}_{0}(\xi)=\hat{\varphi}\left(a_{0} \xi\right) .
$$

We can transport these wavelets and scaling function to the sphere using the inverse stereographic projection, $\Psi_{j l}=\Pi^{-1} \psi_{j l}$ and $\Psi_{0}=\Pi^{-1} \psi_{0}$, and define a CWT discretized in scale and angle as

$$
W f(\omega, j, l)=\int_{S^{2}} \Psi_{j l}\left([\omega]^{-1} \omega^{\prime}\right) f\left(\omega^{\prime}\right) d \omega^{\prime}, \quad j \geq 0
$$

It is now a consequence of our construction that $W f(\omega, j, l)$ can be inverted very simply as

$$
f(\omega)=\sum_{j \geq 0} \sum_{l} W f(\omega, j, l) .
$$

Indeed, the sum in $l$ is taken care of by the angular partition of unity, and the sum in $j$ is telescoping in the sense that

$$
\left[\frac{1}{a_{j}^{2}} \varphi\left(\frac{x}{a_{j}}\right)-\frac{1}{a_{j-1}^{2}} \varphi\left(\frac{x}{a_{j-1}}\right)\right]+\left[\frac{1}{a_{j-1}^{2}} \varphi\left(\frac{x}{a_{j-1}}\right)-\frac{1}{a_{j-2}^{2}} \varphi\left(\frac{x}{a_{j-2}}\right)\right]+\ldots-\frac{1}{a_{0}^{2}} \varphi\left(\frac{x}{a_{0}}\right)=\frac{1}{a_{j}^{2}} \varphi\left(\frac{x}{a_{j}}\right) .
$$

The only remaining term defines an approximation of the identity in the plane, as well as on the sphere after $\Pi^{-1}$. For nice $\varphi$ the convergence of the reconstruction takes place in every $L^{p}$ space, $1 \leq p \leq \infty$. For practical purposes one also discretizes $\omega$ without significant loss of accuracy.

Our advertising claim that inversion can be done in one line of code is true for matrix-friendly languages such as Matlab, where contraction of a 4-dimensional matrix along two of its subscrpits can be done by a double sum instruction.

\footnotetext{
${ }^{\S}$ Through a frame operator, bounded and bounded away from zero.
} 


\section{APPLICATIONS}

The anisotropic continuous wavelet transform introduced in this paper is a very flexible tool for analyzing data defined on $S^{2}$. The spherical CWT has already proved useful, for example in astrophysics. ${ }^{12}$ Gabor wavelets, as defined in this paper, may be of interest for two main reasons. First because they could be more adapted to the fine local geometry of complex datasets (i.e. like for detecting elongated features on the earth's surface). Second because the extra degree of freedom brought by the rotation parameter allows for easily adding redundancy, which could be of interest when one wants to detect objects in very noisy environments. There are also higly promising applications, like for example denoising and studying High Angular Resolution Diffusion datasets for performing efficient fibre tracking. ${ }^{13} \quad$ Spherical Gabor Wavelets also offer a very interesting solution to the problem of star-shaped surface representation. ${ }^{11}$

\section{ACKNOWLEDGMENTS}

L.D. is a fellow of the Francqui Foundation and the Belgian American Educational Foundation.

\section{REFERENCES}

1. J.-P. Antoine, R. Murenzi, and P. Vangergheynst, "Two-dimensional directional wavelets in image processing," Internat. J. Imaging Syst. Technol. 7, pp. 152-165, 1996.

2. E. J. Candès and D. L. Donoho, "New tight frames of curvelets and optimal representation of objects with smooth singularities," Submitted, 2003.

3. P. Frossard, P. Vandergheynst, R. M. Figueras, and M. Kunt, "A posteriori quantization of progressive matching pursuit streams," IEEE Transactions on Signal Processing, To appear, 2003.

4. P. Vandergheynst and P. Frossard, "Efficient image representation by anisotropic refinement in matching pursuit," in Proceedings of IEEE ICASSP, Salt Lake City UT 3, 2001.

5. E. J. Candès and L. Demanet, "Curvelets and fourier integral operators," C. R. Acad. Sci. Paris Ser. I 336, pp. 395-398, 2003.

6. P. Schröder and W. Sweldens, "Spherical wavelets : Efficiently representing functions on the sphere," in ACM Computer Graphics Proc., Proc. SIGGRAPH, pp. 161-175, 1995.

7. J.-P. Antoine and P. Vandergheynst, "Wavelets on the 2-sphere : A group-theoretical approach," Appl. Comput. Harmon. Anal. 7, pp. 262-291, 1999.

8. J.-P. Antoine, L. Demanet, L. Jacques, and P. Vandergheynst, "Wavelets on the sphere : Implementation and approximations," Appl. Comput. Harmon. Anal. 13, pp. 177-200, 2002.

9. W. Freeden, M. Schreiner, and T. Gervens, Constructive Approximation on the Sphere, with applications to Geomathematics, Clarendon Press, Oxford, 1997.

10. S. T. Ali, J.-P. Antoine, and J.-P. Gazeau, Coherent States, Wavelets and Their Generalizations, Springer, New York, 2000.

11. T. Bülow and K. Daniilidis, "Surface representations using spherical harmonics and gabor wavelets on the sphere," Technical Report MS-CIS-01-37, Dept. of Computer and Information Science, University of Pennsylvania, 2001.

12. E. Martinez-Gonzalez, J. Gallegos, F. Argueso, L. Cayon, and J. Sanz, "The performance of spherical wavelets to detect non-gaussianity in the cosmic microwave background sky," Monthly Notices of the Royal Astronomical Society 336, pp. 22-32, October 2002.

13. P. Hagmann, J.-P. Thiran, L. Jonasson, P. Vandergheynst, S. Clarke, P. Maeder, and R. Meuli, "DTI mapping of human brain connectivity: statistical fibre tracking and virtual dissection," Neuroimage 19, July 2003. 